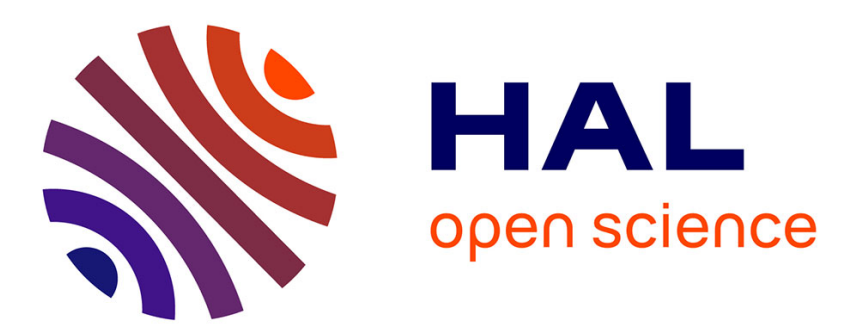

\title{
Effect of the torso conductivity heterogeneities on the ECGI inverse problem solution
}

Nejib Zemzemi, Cecile Dobrzynski, Laura Bear, Mark Potse, Corentin Dallet, Yves Coudière, Remi Dubois, Josselin Duchateau

\section{- To cite this version:}

Nejib Zemzemi, Cecile Dobrzynski, Laura Bear, Mark Potse, Corentin Dallet, et al.. Effect of the torso conductivity heterogeneities on the ECGI inverse problem solution. Computing in cardiology, Sep 2015, Nice, France. hal-01241750

\author{
HAL Id: hal-01241750 \\ https://hal.inria.fr/hal-01241750
}

Submitted on 10 Dec 2015

HAL is a multi-disciplinary open access archive for the deposit and dissemination of scientific research documents, whether they are published or not. The documents may come from teaching and research institutions in France or abroad, or from public or private research centers.
L'archive ouverte pluridisciplinaire HAL, est destinée au dépôt et à la diffusion de documents scientifiques de niveau recherche, publiés ou non, émanant des établissements d'enseignement et de recherche français ou étrangers, des laboratoires publics ou privés. 


\title{
Effect of the torso conductivity heterogeneities on the ECGI inverse problem solution
}

\author{
Nejib Zemzemi ${ }^{1,2,3}$, Cecile Dobrzynski ${ }^{1,3}$, Laura Bear ${ }^{2,4}$, Mark Potse ${ }^{1,2,6}$, \\ Corentin Dallet $^{2,4}$,Yves Coudière ${ }^{1,2,3}$, Remi Dubois ${ }^{2,4}$, and Josselin Duchateau ${ }^{2,4,5}$
}

\begin{abstract}
The effect of torso conductivity heterogeneities on the electrocardiographic imaging (ECGI) inverse problem solution is still subject of debate. In this study we present a method to assess the effect of these heterogeneities. We use an anatomical model containing the heart the lungs the bones and the torso surfaces. We use the bidomain model and we solve it using finite element methods in order to generate in silico data taking into account the torso heterogeneities. We add different noise levels on the body surface potentials and we solve the inverse problem for both homogenous and heterogeneous torso conductivities. We analyse the reconstructed solution using the relative error and the correlation coefficient.
\end{abstract}

\section{INTRODUCTION}

The ECGI technology is a recent approach that helps the cardiologist to visualize the electrical potential distribution or other characteristics of the electrical wave on the heart surface or volume. This procedure is based on 1) electrical measurements on the surface of the torso, 2) some geometrical information obtained from CT-scans or MRI images and 3) some numerical algorithms that allow to solve the inverse problem. The electrical measurements are collected using body surface electrodes. It is well known that the more data you have on the body surface in terms of number of electrodes the better are the results [1], [2]. One should also take into account that these measurements are noisy and have to consider the effect of noise on the inverse solution. The geometrical information is usually constructed using the CTscan images of the patient they allow in particular to extract the torso and heart surfaces. In most of the studies each of the nodes at the torso surface represents an electrode. In this case the torso geometry accuracy depends on the number of electrodes. In some works, if the number of electrodes is too small and not enough to represent the geometry. The mesh could contain nodes that are not located at electrodes positions and in that case there are two options for solving the inverse problem: a) some interpolation techniques could be used in order to predict the electrical potential on these new points, or b) use the refined geometry to construct the transfer operator and then remove the points that do not represent electrodes positions from the inverse reconstruction [2]. The third important element in solving the ECGI

\footnotetext{
${ }^{1}$ Inria Bordeaux Sud-Ouest. Carmen team,

2 IHU LIRYC, Electrophysiology and Heart Modeling Institute Bordeaux, France.

${ }^{3}$ IMB UMR CNRS 5251

${ }^{4}$ CRCTB Inserm U1045. Universit de Bordeaux

${ }^{5}$ Hopital Cadiologique du Haut-Lvque, CHU de Bordeaux, France

${ }^{6}$ CCMC, università della svizzera italiana, switzerland
}

problem is the numerical algorithm. It takes into account the mathematical model representing the forward propagation and all the geometrical electrical data. Few works studied the effect of the torso heterogeneities on the forward solutions [3], [4], [5]. A small number of papers have studied the effect of inhomogeneities on the ECGI inverse solution. In [6] authors worked on analytical solution in concentric sphere. In [7] the author studied the effect of conductivity uncertainties on the inverse solution of ECGI, the study was in 2D and with analytical solutions as well. Here we focus on the effect of combining conductivity heterogeneity and noise in the electrical data on the ECGI solution using 3D realistic geometry.

\section{MethodS}

We use numerical simulations to construct reference data. The torso geometry was constructed from a CT scan of a 43-years-old woman. In order to construct the body surface potentials, we used the bidomain model in the heart and the Laplace equation with heterogeneous conductivities in the torso. After adding different levels of noise on the body surface potentials (BSP), and without filtering, we solved the inverse problem based on an optimal control approach using a zero order Tikhonov regularisation for both homogenous and heterogeneous torso conductivities.

\section{A. Forward problem}

The forward problem is based on the bidomain model that we couple to the torso, details could be found in [9]. The bidomain equations were used to simulate the electrical activity of the heart and extracellular potentials in the whole body. Figure 1 provides a geometrical representation of the domains considered to compute extracellular potentials in the human body. Here we only show the equations governing the electrical wave in the torso domain. Since we suppose that we are in quasi-static condition the electrical potential in the torso domain is obtained by solving the Laplace equation with a Dirichlet boundary condition on the heart surface and a non-flux boundary condition on the body surface. In the torso domain $\Omega_{\mathrm{T}}$, the electrical potential $u_{\mathrm{T}}$ is described by the Laplace equation.

$$
\left\{\begin{array}{rll}
\operatorname{div}\left(\boldsymbol{\sigma}_{\mathrm{T}} \boldsymbol{\nabla} u_{\mathrm{T}}\right)=0, & \text { in } & \Omega_{\mathrm{T}}, \\
u_{\mathrm{T}}=u_{\mathrm{e}}, & \text { on } & \Sigma . \\
\boldsymbol{\sigma}_{\mathrm{T}} \boldsymbol{\nabla} u_{\mathrm{T}} \cdot \boldsymbol{n}_{\mathrm{T}}=0, & \text { on } & \Gamma_{\text {ext }} .
\end{array}\right.
$$

where $\boldsymbol{\sigma}_{\mathrm{T}}$ stands for the torso conductivity tensor and $\boldsymbol{n}_{\mathrm{T}}$ is the outward unit normal to the torso external boundary $\Gamma_{\text {ext }}$. 
Further informations about the finite element methods we

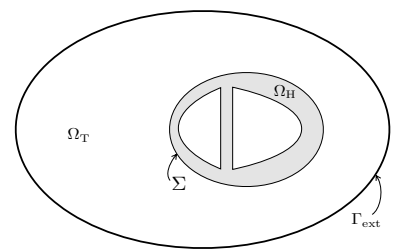

Fig. 1. Two-dimensional geometrical description: heart domain $\Omega_{\mathrm{H}}$, torso domain $\Omega_{\mathrm{T}}$ (extramyocardial regions), heart-torso interface $\Sigma$ and torso external boundary $\Gamma_{\text {ext }}$.

use for solving the bidomain problem coupled to the torso could be found in [10].

\section{B. Anatomical model}

We use a geometry that we have segmented from a CT scan of a 43 year old woman. The DICOM images were segmented using medical imaging softwares. We identify three regions in the torso domain: lungs, bones, and the rest of the torso tissue. After segmentation, we use Tetgen and INRIA meshing Software MMG3D ${ }^{1}$ in order to get a good quality of athetrahedral mesh [11].
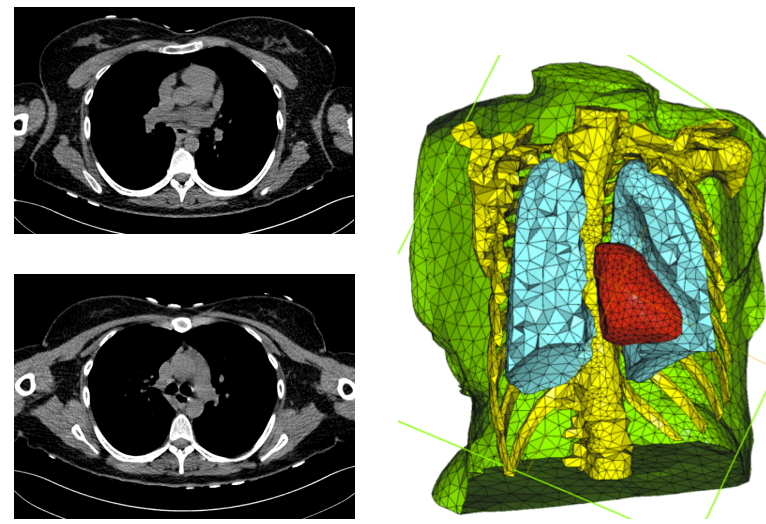

Fig. 2. Two slices of the CT-scan images (left). Torso geometry showing the epicardium (heart-torso interface $\Sigma$ ) (red), lungs (light blue), bones (yellow) and torso external boundary $\Gamma_{\text {ext }}$ (green).

\section{Inverse problem}

ECGI allows to construct the electrical potential on the heart surface $\Sigma$ from data measured on the body surface $\Gamma_{\text {ext }}$. We assume that the electrical potential is governed by the diffusion equation in the torso as shown in the previous paragraph. For a given potential data $T$ measured on the body surface $\Gamma_{\text {ext }}$, the goal is to find $u_{\mathrm{e}}$ on $\Sigma$ such that the potential data in the torso domain satisfies both Dirichlet and Neumann boundary conditions on the epicardium $\Sigma$ as follows

$$
\left\{\begin{array}{r}
\operatorname{div}\left(\boldsymbol{\sigma}_{\mathrm{T}} \boldsymbol{\nabla} u_{\mathrm{T}}\right)=0, \text { in } \Omega_{\mathrm{T}}, \\
\boldsymbol{\sigma}_{\mathrm{T}} \boldsymbol{\nabla} u_{\mathrm{T}} \cdot \boldsymbol{n}=0, \text { and } u_{\mathrm{T}}=T, \text { on } \Gamma_{\mathrm{ext}}, \\
u_{\mathrm{T}}=?, \text { on } \Sigma .
\end{array}\right.
$$

\footnotetext{
${ }^{1}$ http://www.math.u-bordeaux 1.fr/ dobrzyns/logiciels/download.php
}

This problem is ill posed because the $u_{\mathrm{T}}$ is over determined on the boundary $\Gamma_{\text {ext }}$, where we have two boundary conditions to be satisfied. In order to find $u_{\mathrm{T}}$ on the heart surface $\Sigma$, we use an optimal control approach based on the Steklov Poincré variational formulation[12], [13]. This method is inspired from the domain decomposition field. We look for $\tau=u_{\mathrm{T} / \Sigma}$ minimizing the following cost function

$$
J(\lambda)=\frac{1}{2} \int_{\Omega_{\mathrm{T}}}\left(\nabla u_{\mathrm{D}}(\lambda)-\nabla u_{\mathrm{N}}(\lambda)\right)^{2}+\frac{\alpha}{2} \int_{\Sigma} \lambda^{2} .
$$

where $\alpha$ is a regularization parameter and $u_{\mathrm{D}}(\lambda)$ and $u_{\mathrm{N}}(\lambda)$ are solution of the following equations

$$
\begin{array}{r}
\left\{\begin{array}{r}
\operatorname{div}\left(\boldsymbol{\sigma}_{\mathrm{T}} \boldsymbol{\nabla} u_{\mathrm{D}}(\lambda)\right)=0, \text { in } \Omega_{\mathrm{T}}, \\
u_{\mathrm{D}}(\lambda)=T, \text { on } \Gamma_{\mathrm{ext}}, \\
u_{\mathrm{D}}(\lambda)=\lambda, \text { on } \Sigma .
\end{array}\right. \\
\left\{\begin{array}{r}
\operatorname{div}\left(\boldsymbol{\sigma}_{\mathrm{T}} \nabla u_{\mathrm{N}}(\lambda)\right)=0, \text { in } \Omega_{\mathrm{T}}, \\
\boldsymbol{\sigma}_{\mathrm{T}} \boldsymbol{\nabla} u_{\mathrm{N}}(\lambda) \cdot \boldsymbol{n}=0, \text { on } \Gamma_{\mathrm{ext}}, \\
u_{\mathrm{N}}(\lambda)=\lambda, \text { on } \Sigma .
\end{array}\right.
\end{array}
$$

Since we are interested in assessing the effect of torso conductivity inhomogeneities on the ECGI inverse solution, the conductivity function $\boldsymbol{\sigma}_{\mathrm{T}}$ would be constant when we are in the homogeneous case and depends on the considered regions (lungs, bones and remaining tissue) in the heterogeneous case. Details about the iterative algorithm solving the minimization problem could be found in [12].

\section{NUMERICAL RESULTS}

We start by generating synthetic data using an ECG simulator based on the bidomain model. We stimulate the heart at the apex, the electrical wave propagates from apex to base. In figure 3 , we show the distribution of the electrical potential in the depolarization phase. In figure 4 , we show the potential distribution at the repolarization phase.
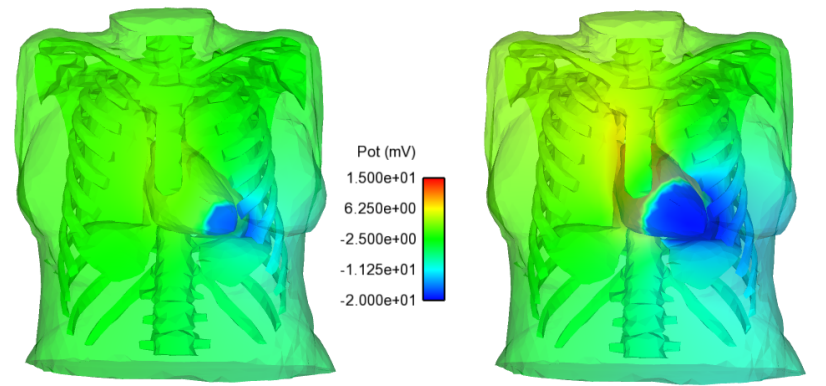

Fig. 3. Depolarization phase: Snapshots of potential distribution on the heart surface and the torso volume at times $16 \mathrm{~ms}$ (left) and $40 \mathrm{~ms}$ (right). The color bar scale is in $\mathrm{mV}$.

\section{A. Zero noise}

From the forward solution, we extract the electrical potential at the body surface and we compute the inverse solution without adding noise. In figure 5, we show the relative error for the homogeneous (blue) and inhomogeneous (red) cases when no noise is considered. In the heterogeneous 

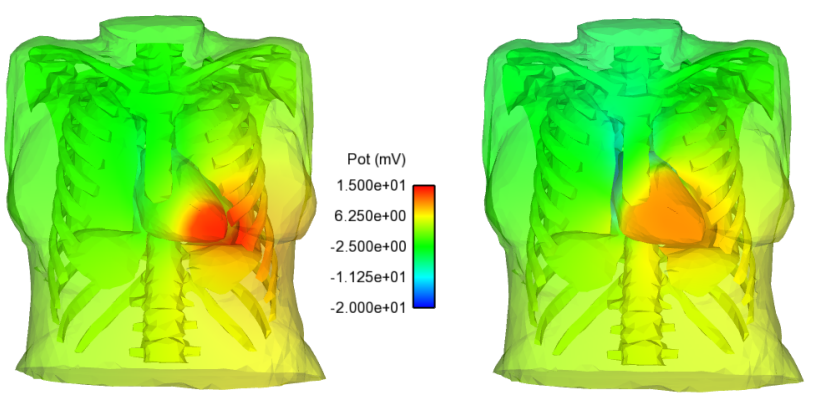

Fig. 4. Repolarization phase: Snapshots of potential distribution on the heart surface and the torso volume at times $176 \mathrm{~ms}$ (left) and $224 \mathrm{~ms}$ (right). The color bar scale is in $\mathrm{mV}$.

(respectively, homogeneous) case, the mean value of the RE in time is 0.38 (respectively, 0.52) and its standard deviation is 0.05 (respectively, 0.04).

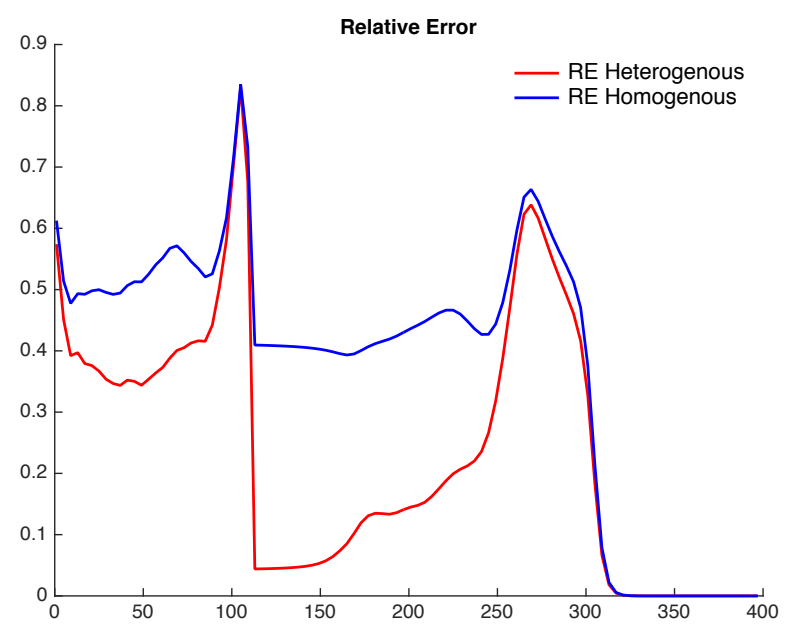

Fig. 5. Time course of the relative error between the forward and the inverse solution for the heterogeneous conductivity case (red) and the homogeneous case (blue). No noise is added to the BSPs.

In figure 6, we show the correlation coefficient for the homogeneous (blue) and inhomogeneous (red) for the same case. In the heterogeneous (respectively, homogeneous) case, the mean value of the $\mathrm{CC}$ in time is 0.92 (respectively, 0.86) and its standard deviation is 0.024 (respectively, 0.026).

\section{B. Effect of noise}

We add different levels of noise to the extracted body surface potentials, the amplitude of the noise increase from $0.5 \mathrm{mV}$ to $1.0 \mathrm{mV}$ and $2.0 \mathrm{mV}$. An example of Body surface potential time course extracted from a point on the torso surface is given in figure 7 . We solve the inverse problem using the noisy BSP data and we compute the RE and the CC in the depolarization interval (between $0 \mathrm{~ms}$ and $80 \mathrm{~ms}$ ). For the heterogeneous case, adding a noise of amplitude $0.5 \mathrm{mV}$ (respectively $1.0 \mathrm{mV}, 2.0 \mathrm{mV}$ ) comes with a relative error of $0.46 \pm 0.11$ (respectively, $0.49 \pm 0.1,0.56 \pm 0.13$ ). The $\mathrm{CC}$ decreases from $0.92 \pm 0.02$ to $0.90 \pm 0.03$ (respectively, $0.87 \pm 0.07,0.80 \pm 0.12$ ).

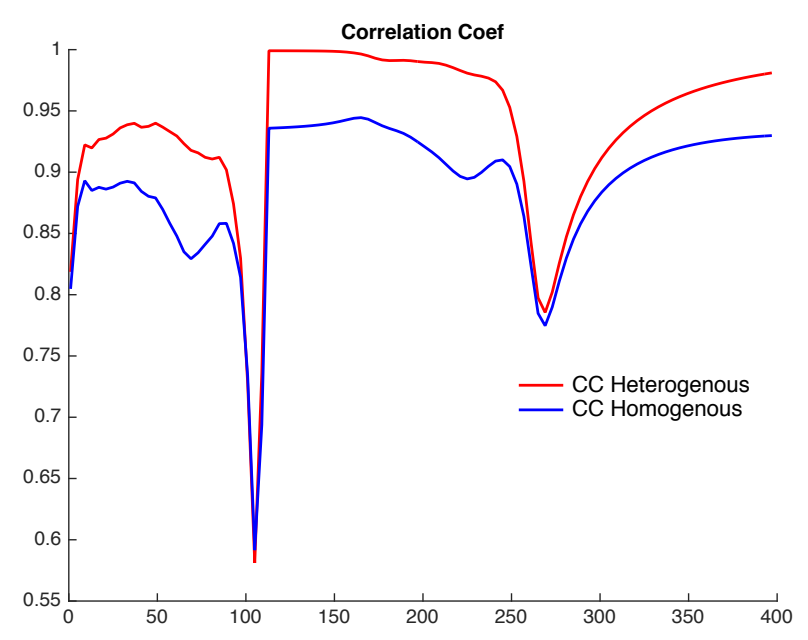

Fig. 6. Time course of the correlation coefficient between the forward and the inverse solution for the heterogeneous conductivity case (red) and the homogeneous case (blue). No noise is added to the BSPs

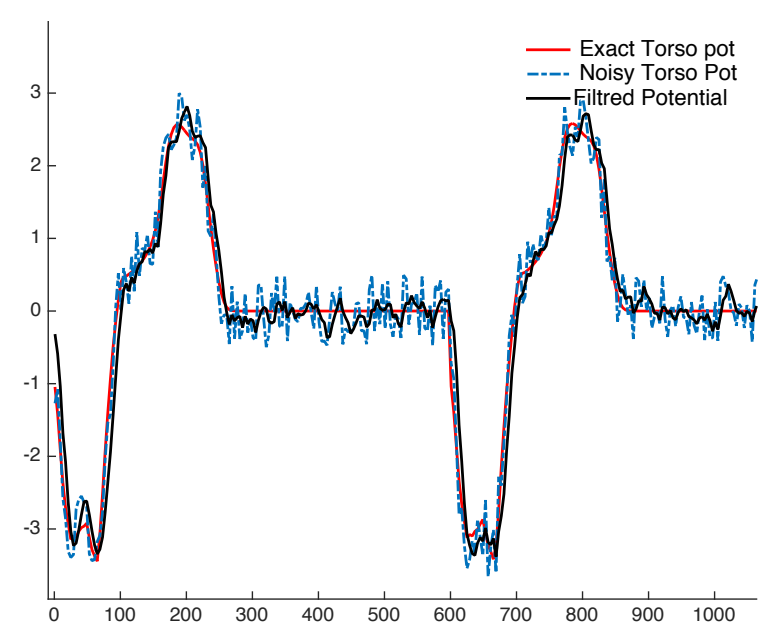

Fig. 7. Example of BSP at a given point at the torso surface: exact potential (red), noisy potential by adding $0.5 \mathrm{mV}$ (bleu dashed line) and filtered potential (black continuous line).

For the homogeneous case, adding a noise of amplitude 0.5 $\mathrm{mV}$ (respectively $1.0 \mathrm{mV}, 2.0 \mathrm{mV}$ ) comes with a relative error of $0.57 \pm 0.07$ (respectively, $0.59 \pm 0.07,0.63 \pm 0.10$ ). The $\mathrm{CC}$ decreases from $0.86 \pm 0.02$ to $0.85 \pm 0.03$ (respectively, $0.82 \pm 0.07,0.77 \pm 0.11$ ). As an example, we show in figure 8 , the time course of the $\mathrm{CC}$ and in figure 9 the RE for the case where we add a noise of amplitude $0.5 \mathrm{mV}$ for both homogeneous and heterogeneous torso. We see that the gap between the homogeous and heterogenous case both in terms of $\mathrm{RE}$ and $\mathrm{CC}$ is reduced by the noise.

\section{Discussion}

We have presented a study of the effect of the heterogeneities in the torso conductivity on the ECGI inverse solution. Since we were using numerical simulations, we were able to construct an ideal experiment where we isolated the effect of the heterogeneities. All the other factors like geometry of heart and torso and their positions, BSPs, ground 


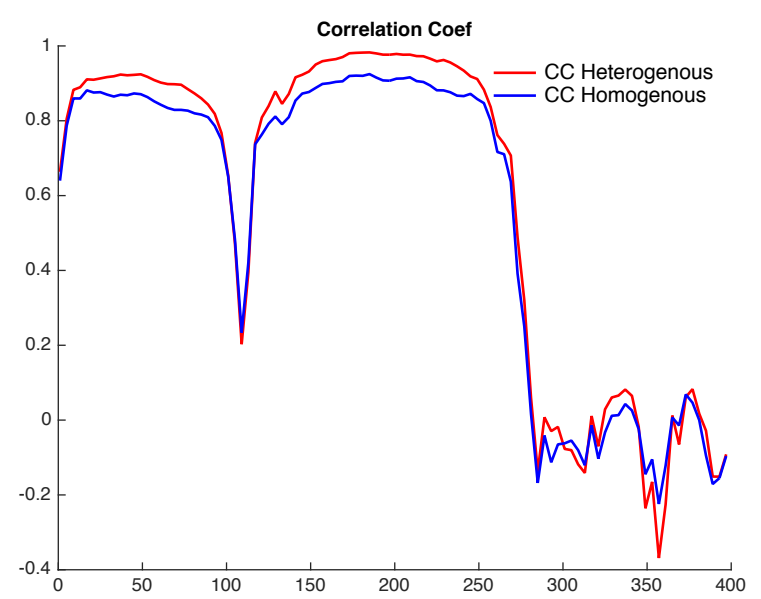

Fig. 8. Time course of the correlation coefficient between the forward and the inverse solution for the heterogeneous conductivity case (red) and the homogeneous case (blue). Here we add a noise of magnitude $0.5 \mathrm{mV}$ to the BSPs.

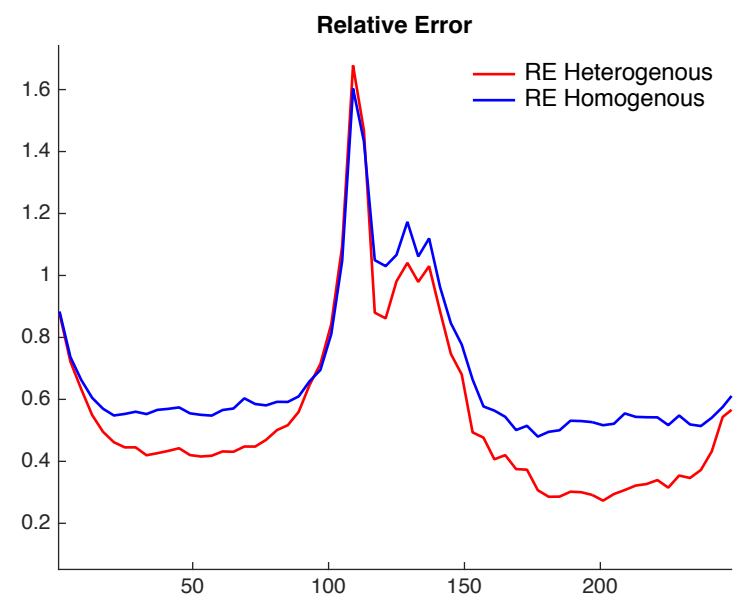

Fig. 9. Time course of the RE between the forward and the inverse solution for the heterogeneous conductivity case (red) and the homogeneous case (blue). Here we add a noise of magnitude $0.5 \mathrm{mV}$ to the BSPs.

truth electrograms,..., etc are the same.

Our results show that for low values of noise in the BSP measurements, the effect of torso heterogeneities is clear: there is a gain between 5 to $10 \%$ of accuracy in terms of $\mathrm{CC}$ and 10 to $20 \%$ in terms of RE if these heterogeneities are accounted for in the inverse model. This gain decreases when the noise in the BSP measurement increases. The correlation coefficient is too small and even negative between $270 \mathrm{~ms}$ and $400 \mathrm{~ms}$ in figure 8 . This is mainly du to the fact that the heart is fully repolarized and the torso potential is too small. In this case the noise is relatively high compared to the values of the potential. The inverse solution is capturing only the noise. That is why in figure 9 we only show the RE between 0 and $270 \mathrm{~ms}$.

\section{CONCLUSION}

In this paper, we presented a numerical study showing the effect of the torso conductivity inhomogeneities on the ECGI inverse solution. We formulated the ECGI inverse problem in terms of electrograms and we used an optimal control approach to solve it. We have considered a 3D realistic geometry of a 43 years old women. The geometry contains the lungs bones and the remaining tissue domains. We considered the heterogeneous for building the gold standard data. And we solved the inverse problem using homogeneous and inhomogeneous torso models. Our results show that high levels of noise hide the effect of the heterogeneity on the inverse solution. But when the noise level is relatively small, we have seen that considering the heterogeneities in the torso conductivity improves the quality of the inverse solution in terms of $\mathrm{CC}$ by 5 to $10 \%$ and RE by 10 to $20 \%$. This experiment would be very difficult to be performed in in vivo experiments since the noise in the measured data and the experiment environement would highly interfere with the effect of the conductivities as shown in our results.

\section{ACKNOWLEDGEMENT}

This work was supported by the Agence Nationale de la Recherche (Grant IHU LIRYC ANR-10-IAHU-04)

\section{REFERENCES}

[1] Ghosh S, Rudy Y. Accuracy of quadratic versus linear interpolation in noninvasive electrocardiographic imaging (ecgi). Annals of biomedical engineering 2005;33(9):1187-1201.

[2] Ghodrati A, Brooks DH, MacLeod RS. Methods of solving reduced lead systems for inverse electrocardiography. Biomedical Engineering IEEE Transactions on 2007;54(2):339-343.

[3] Geneser SE, Kirby RM, MacLeod RS. Application of stochastic finite element methods to study the sensitivity of ecg forward modeling to organ conductivity. Biomedical Engineering IEEE Transactions on 2008;55(1):31-40.

[4] Van Oosterom A, Huiskamp G. The effect of torso inhomogeneities on body surface potentials quantified using tailored geometry. Journal of electrocardiology 1989;22(1):53-72.

[5] Weber FM, Keller DU, Bauer S, Seemann G, Lorenz C, Dossel O. Predicting tissue conductivity influences on body surface potentialsan efficient approach based on principal component analysis. Biomedical Engineering IEEE Transactions on 2011;58(2):265-273.

[6] Throne RD, Olson LG. The effects of errors in assumed conductivities and geometry on numerical solutions to the inverse problem of electrocardiography. Biomedical Engineering IEEE Transactions on 1995;42(12):1192-1200.

[7] Aboulaich R, Fikal N, El Guarmah E, Zemzemi N. Sensitivity of the electrocardiography inverse solution to the torso conductivity uncertainties. In Functional Imaging and Modeling of the Heart. Springer, 2015; 475-483.

[8] Zemzemi N. Étude théorique et numérique de l'activité électrique du cœur: Applications aux électrocardiogrammes. Ph.D. thesis, Université Paris XI, 2009. http://tel.archives-ouvertes.fr/ tel-00470375/en/.

[9] Zemzemi N, Bernabeu M, Saiz J, Rodriguez B. Simulating DrugInduced Effects on the Heart: From Ion Channel to Body Surface Electrocardiogram. Functional Imaging and Modeling of the Heart 2011;259-266.

[10] Boulakia M, Cazeau S, Fernández M, Gerbeau J, Zemzemi N. Mathematical modeling of electrocardiograms: a numerical study. Annals of biomedical engineering 2010;38(3):1071-1097. ISSN 0090-6964.

[11] Dapogny C, Dobrzynski C, Frey P. Three-dimensional adaptive domain remeshing, implicit domain meshing, and applications to free and moving boundary problems. Journal of Computational Physics 2014;262(0):358 - 378. ISSN 0021-9991.

[12] Zemzemi N. A steklov-poincaré approach to solve the inverse problem in electrocardiography. In Computing in Cardiology Conference (CinC), 2013. IEEE, 2013; 703-706.

[13] Belgacem FB, El Fekih H. On cauchy's problem: I. a variational steklov-poincaré theory. Inverse Problems 2005;21(6):1915. 